\title{
High-resolution hard X-ray and gamma-ray spectrometers based on superconducting absorbers coupled to superconducting transition edge sensors
}

\author{
M. L. van den Berg, D. T. Chow, A. Loshak, \\ M. F. Cunningham, T. W. Barbee Jr., M. Frank, \\ and S. E. Labov
}

This article was submitted to

SPIE Meeting

San Diego, CA

July30 - August 4, 2000

U.S. Department of Energy

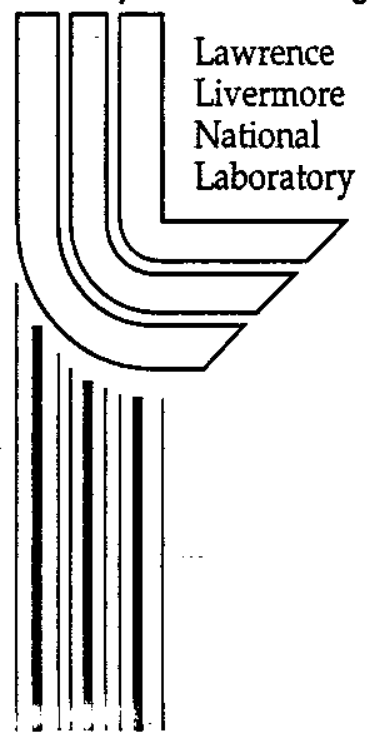

September 21, 2000 


\section{DISCLAIMER}

This document was prepared as an account of work sponsored by an agency of the United States Government. Neither the United States Government nor the University of California nor any of their employees, makes any warranty, express or implied, or assumes any legal liability or responsibility for the accuracy, completeness, or usefulness of any information, apparatus, product, or process disclosed, or represents that its use would not infringe privately owned rights. Reference herein to any specific commercial product, process, or service by trade name, trademark, manufacturer, or otherwise, does not necessarily constitute or imply its endorsement, recommendation, or favoring by the United States Government or the University of California. The views and opinions of authors expressed herein do not necessarily state or reflect those of the United States Government or the University of California, and shall not be used for advertising or product endorsement purposes.

This is a preprint of a paper intended for publication in a journal or proceedings. Since changes may be made before publication, this preprint is made available with the understanding that it will not be cited or reproduced without the permission of the author.

This report has been reproduced directly from the best available copy.

Available to DOE and DOE contractors from the Office of Scientific and Technical Information

P.O. Box 62, Oak Ridge, TN 37831

Prices available from (423) 576-8401 http://apollo.osti.gov/bridge/

Available to the public from the National Technical Information Service

U.S. Department of Commerce 5285 Port Royal Rd., Springfield, VA 22161 http://www.ntis.gov/

OR

Lawrence Livermore National Laboratory Technical Information Department's Digital Library http://www.llnl.gov/tid/Library.html 


\section{DISCLAIMER}

This document was prepared as an account of work sponsored by an agency of the United States Government. Neither the United States Government nor the University of California nor any of their employees, makes any warranty, express or implied, or assumes any legal liability or responsibility for the accuracy, completeness, or usefulness of any information, apparatus, product, or process disclosed, or represents that its use would not infringe privately owned rights. Reference herein to any specific commercial product, process, or service by trade name, trademark, manufacturer, or otherwise, does not necessarily constitute or imply its endorsement, recommendation, or favoring by the United States Government or the University of California. The views and opinions of authors expressed herein do not necessarily state or reflect those of the United States Government or the University of California, and shall not be used for advertising or product endorsement purposes.

This is a preprint of a paper intended for publication in a journal or proceedings. Since changes may be made before publication, this preprint is made available with the understanding that it will not be cited or reproduced without the permission of the author.

This report has been reproduced

directly from the best available copy.

Available to DOE and DOE contractors from the

Office of Scientific and Technical Information

P.O. Box 62, Oak Ridge, TN 37831

Prices available from (423) 576-8401

http://apollo.osti.gov/bridge/

Available to the public from the

National Technical Information Service

U.S. Department of Commerce

5285 Port Royal Rd.,

Springfield, VA 22161

http://www.ntis.gov/

OR

Lawrence Livermore National Laboratory

Technical Information Department's Digital Library

http://www.llnl.gov/tid/Library.html 
Presented at SPIE conference on X-ray and Gamma-ray Instrumentation for Astronomy XI, San Diego, 2000. To be published in Proc. SPIE 4140, 2000.

\title{
High-resolution hard X-ray and gamma-ray spectrometers based on superconducting absorbers coupled to superconducting transition edge sensors
}

\author{
M.L. van den $\operatorname{Berg}^{*} a$, D.T. Chow ${ }^{a, b}$, A. $\operatorname{Loshak}^{a}$, M.F. Cunningham ${ }^{a, b}$, T.W. Barbee Jr. ${ }^{a}$, \\ M. Frank ${ }^{a}$, S.E. Labov ${ }^{a}$ \\ ${ }^{a}$ Lawrence Livermore National Laboratory, Livermore, CA, 94550 \\ ${ }^{b}$ Department of Applied Science, University of California, Davis
}

\begin{abstract}
We are developing detectors based on bulk superconducting absorbers coupled to superconducting transition edge sensors (TES) for high-resolution spectroscopy of hard X-rays and soft gamma-rays. We have achieved an energy resolution of $70 \mathrm{eV} \mathrm{FWHM} \mathrm{at} 60 \mathrm{keV}$ using a $1 \times 1 \times 0.25 \mathrm{~mm}^{3} \mathrm{Sn}$ absorber coupled to a Mo/Cu multilayer TES with a transition temperature of $100 \mathrm{mK}$. The response of this detector is compared with a simple model using only material properties data and characteristics derived from IV-measurements. We have also manufactured detectors using superconducting absorbers with a higher stopping power, such as $\mathrm{Pb}$ and $\mathrm{Ta}$. We present our first measurements of these detectors, including the thermalization characteristics of the bulk superconducting absorbers. The differences in performance between the detectors are discussed and an outline of the future direction of our detector development efforts is given.
\end{abstract}

Keywords: microcalorimeter, superconducting absorbers, x-ray spectroscopy, gamma-ray spectroscopy

\section{INTRODUCTION}

Hard X-ray and soft gamma-ray spectrometers with a high energy resolution ( $E / \Delta E \gtrsim 1000$ between $20-500 \mathrm{keV})$ are of great interest to many fields. High-resolution gamma-ray spectrometers are an important inspection tool for nuclear weapons material. A high spectral resolution enables a precise determination of the level of enrichment in nuclear material and therefore provides increased sensitivity and selectivity in searching for these materials. In high-energy astrophysics, a high-energy resolution spectrometer would be very useful for the detection of ${ }^{44} \mathrm{Ti}$ decay (67.8 and $78.3 \mathrm{keV}$ ) in the universe as well as for studying accreting neutron stars and black holes. Other applications are experimental studies of fundamental physics, such as Lamb shift measurements of high- $Z$ atoms and Compton scattering experiments, but also environmental monitoring. By analyzing uranium radiation in ocean water samples it can be determined when the water has last been in contact with land. For this type of analysis, a high-resolution spectrometer is required to isolate the ${ }^{235} \mathrm{U}$ radiation at $185.7 \mathrm{keV}$ from the ${ }^{226} \mathrm{Ra}$ background radiation $(186.1 \mathrm{keV})$.

Currently the state-of-the-art gamma-ray detector for high-resolution spectroscopy is a high-purity germánium ionization detector. The resolution of a germanium ionization spectrometer, however, is limited to $\sim 300 \mathrm{eV}$ FWHM at $60 \mathrm{keV}$ by the statistical fluctuation in the number of electron-hole pairs that are created by the gamma-ray photon. A vast improvement can be made by using a low temperature calorimeter. The energy resolution of these devices is limited by the thermodynamic temperature fluctuations in the detector. Depending on the absorber dimensions and the operating temperature, the energy resolution can be one to two orders of magnitude better than achievable with germanium ionization spectrometers.

\footnotetext{
${ }^{*}$ Correspondence: E-mail: MvandenBerg@llnl.gov
} 


\section{PRINCIPLE OF OPERATION}

A microcalorimeter consists of an absorber, with heat capacity $C_{\mathbf{a b s}}$, and a thermometer. The calorimeter is thermally linked with a conductivity $G_{\text {bath }}$ to a heat bath at temperature $T_{\text {bath }}$ (typically less than 0.1 Kelvin). When a photon hits the absorber, the temperature increases. The thermometer simply measures this increase in temperature. The maximum count rate is limited by the thermal time constant $\tau=C_{\text {tot }} / G_{\text {bath }}$, where $C_{\text {tot }}$ is the total heat capacity of the detector. The energy resolution of a calorimeter is intrinsically limited by statistical fluctuations of the detector temperature ${ }^{1}$ :

$$
\Delta E_{\mathrm{FWHM}} \approx 2.355 \sqrt{k_{B} T^{2} G_{\mathrm{bath}} / 4 B}
$$

with $k_{B}$ Boltzmann's constant, $T$ the detector temperature and $B$ the noise bandwidth of the electronic or digital (shaping) filter. When the signal is autocorrelated, the optimum signal to noise ratio is obtained. Assuming the gamma-ray signal is exponentially decaying with time constant $\tau=C_{\text {tot }} / G_{\text {bath }}$, the noise bandwidth of the autocorrelation filter is given by:

$$
B=\frac{1}{4 \tau}
$$

thus yielding for Equation (1):

$$
\Delta E_{\mathrm{FWHM}} \approx 2.355 \sqrt{k_{B} T^{2} C_{\mathrm{tot}}}
$$

As a thermometer we use a superconducting film that is operated at the phase transition between the normal and superconducting state. Such a sensor is commonly called a superconducting-normal phase transition edge sensor or TES. A TES is an extremely sensitive thermometer: The relative change of the resistance with temperature $(\alpha \equiv T / R \cdot d R / d T)$ can be as high as 1000 .

The high responsivity of the TES allows the device to be operated in strong electrothermal feedback mode. ${ }^{2}$ The difference between normal operation (using a current bias) and operation with electrothermal feedback is that the detector is Joule-heated by a voltage bias across the TES to a higher temperature than the heat bath. When a gamma-ray photon heats up the absorber, the TES resistance increases and consequently the Joule-heating is decreased. The heat pulse from an absorbed gamma-ray photon is compensated by a decrease in electrical power input (electrothermal feedback). The feedback decreases the response time of the detector by a factor $\approx n / \alpha$, where $n$ is a constant between 4 and 6 , depending on the heat loss process to the substrate. The faster response also improves the intrinsic energy resolution, since the noise bandwidth of the optimum filtering (autocorrelation) is inversely proportional to the characteristic decay time. ${ }^{2}$

\section{DETECTOR DESIGN}

Low temperature calorimeters have the ability to combine both an excellent energy resolution and a reasonable effective area. Our aim is to build a detector which combines a high resolution (100 eV FWHM at $100 \mathrm{keV}$ and 200 eV FWHM at $500 \mathrm{keV}$ ) with a reasonable quantum efficiency ( $>10 \%$ at $500 \mathrm{keV})$ and area $\left(4 \mathrm{~mm}^{2}\right.$ per pixel) that can be operated at bath temperatures $T_{\text {bath }} \gtrsim 65 \mathrm{mK}$. In order to achieve these goals we make use of a composite detector consisting of an absorber glued to a superconductive transition edge sensor (TES).

\subsection{Transition edge sensor}

The transition edge sensor consists of a Mo/Cu multilayer thin film, which forms a superlattice. The $T_{\mathrm{c}}$ can be tuned by varying the $\mathrm{Mo} / \mathrm{Cu}$ thickness ratio. In our present TES microcalorimeter, the thickness of the Mo layer is $20 \AA$ and the $\mathrm{Cu}$ layer is $70 \AA$ thick. This ratio gives a $T_{\mathrm{c}} \approx 100 \mathrm{mK}$. The TES film consists of 23 Cu layers and 20 Mo layers, giving a total thickness of $200 \mathrm{~nm}$.

A multilayer TES has several potential advantages over the commonly used bilayer TES. The density of states in a bilayer varies over the length scale of the proximity effect. Consequently, the current density cannot be made $^{\prime}$ arbitrarily small in a bilayer. The density of states in a superlattice is more uniform and the current flow is not limited by the proximity effect, but by the thickness of the film. Since the density of states is related to the sharpness of the transition curve, $\alpha$ can be much higher in multilayers. Furthermore, the current density in the multilayers can be made arbitrarily low, thereby enabling operation at low resistance (higher $\alpha$ ) while keeping the bias current high. 


\subsection{Superconducting absorbers}

To obtain a high energy resolution the total heat capacity of the detector should be as small as possible. The heat capacity will be dominated by the absorber since a large pixel area and high quantum efficiency requires a considerable volume. The choice of absorber materials is limited to insulators, semiconductors and superconductors, because the electronic heat capacity of normal metals (and semi-metals) is too high for our application. Since the phonon heat capacity decreases strongly with temperature $\left(\propto T^{3}\right)$, the intrinsic energy resolution for these materials improves significantly with decreasing temperature $\Delta E \propto T^{5 / 2}$. Insulators and semiconductors have the disadvantage that part of the energy of a gamma-ray photon will be dissipated by electrons in long-living states or traps with a relatively high energy $(\gtrsim 1 \mathrm{eV})$. Variation in the number of excited electrons in these materials causes a degradation of energy resolution. ${ }^{3}$

The lifetime of the electronic excitations (quasiparticles) in a superconductor is also very long. Since two quasiparticles are required for recombination into a Cooper pair, the quasiparticle life time is inversely proportional to the quasiparticle density. ${ }^{4}$ Furthermore, the phonon that is produced upon recombination has a significant chance of breaking up another Cooper pair (phonon trapping). The effective lifetime of the quasiparticle excitations in a superconducting absorber therefore depends on many factors, such as the number of excited quasiparticles, the speed of the quasiparticle diffusion, the absorber dimensions and the phonon decay time or escape time. For a temperature $T \ll T_{\mathrm{c}}$ and an absorber volume of $0.25 \mathrm{~mm}^{3}$, the expected quasiparticle life time is typically much larger than 1 second.

So as with semiconductors and insulators, the energy of a photon that is absorbed in a superconductor is divided between the phonons and long-living electronic excitations. However, because the quasiparticle excitation energy is very small $(\sim \mathrm{meV})$, the statistical variation in the number of excited states (quasiparticles) is much less in a superconductor than in insulators and semiconductors. A superconducting absorber is therefore less sensitive to resolution degradation due to statistical variation associated with incomplete thermalization. ${ }^{3}$

The fraction of the incoming energy that is converted into phonons depends on more factors than just the statistical variation in the number of excited quasiparticles. It is also sensitive to variations in the quasiparticle life time. A shorter quasiparticle life time will cause an increased phonon signal. Because the quasiparticle life time is inversely proportional to the quasiparticle density, the actual quasiparticle density will vary from pulse to pulse if the quasiparticle life time is longer than the average time between photon absorptions. In order to obtain a good energy resolution, it is essential that the thermalization rate is faster than the photon count rate.

Vitale et al. ${ }^{3,5}$ have carried out an extensive study on the thermalization properties of several superconducting monocrystals with a volume of about $1 \mathrm{~mm}^{3}$. They found a universal dependence of the thermalization time on $T / \Theta_{\mathrm{D}}$, with $\Theta_{\mathrm{D}}$ the Debye temperature. For temperatures $T>2 \times 10^{-4} \Theta_{\mathrm{D}}$ the thermalization was fast $(<20 \mathrm{~ms})$ and complete. Two microcrystalline samples showed a significantly faster thermalization time. Several other groups have measured the thermalization time of superconducting absorbers and values between $1 \mathrm{~ms}$ and $600 \mathrm{~ms}$ have been reported in the literature. ${ }^{6-9}$

Because the achievable energy resolution depends on so many factors, we have started a study to optimize superconducting absorbers for our particular detector application, which requires a high stopping power for photons with an energy between 100 and $500 \mathrm{keV}$. We use thick high-purity foils in order to obtain a fast thermalization. Table 1 shows the properties of the most interesting superconductor materials. In addition to the critical temperature $T_{\mathrm{c}}$ and the Debye temperature $\Theta_{\mathrm{D}}$, the phonon heat capacity at $100 \mathrm{mK}$ and the penetration depth at $100 \mathrm{keV}$ $\left(\mu_{100 \mathrm{keV}}^{-1}\right)$ is given. The last column shows the phonon noise limited energy resolution without electrothermal feedback, Equation (3), of a $1 \mathrm{~mm}^{2}$ absorber with a thickness equal to three times the penetration depth $\mu_{100}^{21} \mathrm{keV}$ as required for a $95 \%$ absorption efficiency at $100 \mathrm{keV}$.

Clearly, the most interesting materials are Re, Ta and Mo. However, as other properties might limit the achievable energy resolution, $\mathrm{Pb}$ and $\mathrm{Sn}$ are also included in the study. We have started our detector development by optimizing and understanding TES microcalorimeters with Sn absorbers, ${ }^{10}$ because other groups have achieved excellent results using $\mathrm{Sn}$ as an absorber for X-ray microcalorimeters. ${ }^{11-13}$

\subsection{Implementation}

Figure 1a shows a schematic diagram of the gamma-ray detector. The TES multilayer and aluminum superconducting leads are sputter-deposited and structured on a $0.5 \mu \mathrm{m}$ thick silicon-nitride membrane using standard lift-off 
Table 1. Most important characteristics of superconductors that are of interest as an absorber for a gamma-ray calorimeter. $T_{\mathrm{c}}$ is the superconducting transition temperature, $\theta_{\mathrm{D}}$ the Debye temperature, $C_{\text {phonon }}$ the phonon heat capacity and $\mu_{100 \mathrm{keV}}^{-1}$ the $1 / e$ photon absorption depth at $100 \mathrm{keV}$. The last column indicates the phonon noise contribution to the energy resolution assuming no electrothermai feedback, Equation (3), for a 1 mm² absorber at $100 \mathrm{mK}$ with a thickness that is sufficient to absorb $95 \%$ of the incoming photons at $100 \mathrm{keV}$.

\begin{tabular}{|c|c|c|c|c|c|}
\hline & $\begin{array}{r}T_{\mathrm{c}} \\
(\mathrm{K})\end{array}$ & $\begin{array}{c}\Theta_{\mathrm{D}} \\
(\mathrm{K})\end{array}$ & $\begin{array}{c}C_{\text {phonon }}(\mathrm{T}=100 \mathrm{mK}) \\
\left(\mathrm{keV} / \mathrm{mK} / \mathrm{mm}^{3}\right)\end{array}$ & $\begin{array}{c}\mu_{100 \mathrm{keV}}^{-1} \\
(\mathrm{~mm})\end{array}$ & $\begin{array}{c}\Delta E_{\mathrm{FWHM}} \\
(\mathrm{eV})\end{array}$ \\
\hline \hline $\mathrm{Mo}$ & 0.9 & 460 & 13 & 1.02 & 14 \\
\hline $\mathrm{Sn}$ & 3.7 & 195 & 101 & 1.58 & 48 \\
\hline $\mathrm{Ta}$ & 4.5 & 258 & 65 & 0.15 & 12 \\
\hline $\mathrm{Re}$ & 1.7 & 430 & 17 & 0.13 & 6 \\
\hline $\mathrm{Pb}$ & 7.2 & 105 & 574 & 0.17 & 38 \\
\hline
\end{tabular}

photolithography. The superconducting absorber is glued on the thin film device with Stycast 2850FT' epoxy, using an XYZ stage with vacuum tweezers. The diameter of the Stycast is approximately $100 \mu \mathrm{m}$, and the thickness 25 $\mu \mathrm{m}$. The heat capacity of the Stycast is estimated to be $C_{\text {Stycast }}=2 \mathrm{keV} / \mathrm{mK}$ at $\mathrm{T}=100 \mathrm{mK} .^{14}$

The total heat capacity is generally dominated by the heat capacity of the superconducting absorber. For a typical absorber volume of $0.25 \mathrm{~mm}^{3}, C_{\mathrm{abs}}$ varies between $3 \mathrm{keV} / \mathrm{mK}$ for a Mo and $140 \mathrm{keV} / \mathrm{mK} \mathrm{for} \mathrm{a} \mathrm{Pb}$ absorber at $\mathrm{T}=100 \mathrm{mK}$. The Stycast between the absorber and the TES film forms a bottleneck, which reduces the sensitivity of the TES to the non-equilibrium phonons in the superconducting absorber. Taking $R_{\mathrm{Kapitza}}=6.2 / 7^{3} \mathrm{~K}^{4} \cdot \mathrm{cm}^{2} / \mathrm{W}$ as measured for the Kapitza boundary resistance between $\mathrm{Cu}$ and an epoxy glue (Epibond 121) as a typical value, ${ }^{15}$ one obtains for the Kapitza resistance at the two glue surfaces $G_{\text {Kapitza }}=13 \mathrm{nW} / \mathrm{K}$ at $T=100 \mathrm{mK}$. The thermal conductivity of the Stycast is of the same order of magnitude: $G_{\text {Stycast }}=6 \mathrm{nW} / \mathrm{K}$ at $\mathrm{T}=100 \mathrm{mK}{ }^{16}$

The membrane and superconducting leads provide the weak thermal link between the TES and the cold bath. The thermal conductivity $G_{\text {bath }}$ is dominated by the silicon-nitride membrane. The thermal conductivity can be derived from the IV curves of the TES at different bath temperatures. ${ }^{10}$ For $T_{\mathrm{c}}=100 \mathrm{mK}$ and $T_{\text {bath }}=70 \mathrm{mK}$, $G_{\text {bath }} \sim 0.3 \mathrm{nW} / \mathrm{K}$.

The coupling between the electrons and phonons in the multilayer is very good: $G_{\mathrm{e}-\mathrm{p}} \sim 100 \mathrm{nW} / \mathrm{K}$ at $T=$ $100 \mathrm{mK} .^{17}$ Since the electron heat capacity in the TES $C_{\text {TES-e }} \sim 10 \mathrm{keV} / \mathrm{mK}$ is much larger than the phonon heat capacity $C_{\text {TES-p }} \sim 10^{-3} \mathrm{keV} / \mathrm{mK}$, the phonon temperature follows the electron temperature within a few nanoseconds, so the electron-phonon coupling does not play any role in the detector performance.

\section{THEORETICAL RESPONSE}

The microcalorimeter response can be modeled straightforwardily by a set of linear differential equations based on the thermal circuit, ${ }^{18}$ shown in Fig. 1b:

$$
\begin{aligned}
\frac{d T_{\text {abs }}}{d t} & =-\frac{G_{\text {Kapitza+Stycast }}}{C_{\text {abs }}}\left(T_{\text {abs }}-T_{\text {Stycast }}\right) \\
\frac{d T_{\text {Stycast }}}{d t} & =-\frac{G_{\text {Kapitza+Stycast }}}{C_{\text {Stycast }}}\left(T_{\text {Stycast }}-T_{\text {TES-p }}\right)+\frac{G_{\text {Kapitza+Stycast }}}{C_{\text {Stycast }}}\left(T_{\text {Abs }}-T_{\text {Stycast }}\right) \\
\frac{d T_{\text {TES-p }}}{d t} & =-\frac{G_{\text {e-p }}}{C_{\text {TES-p }}}\left(T_{\text {TES-p }}-T_{\text {TES-e }}\right)-\frac{G_{\text {bath }}}{C_{\text {TES-p }}}\left(T_{\text {TES-p }}-T_{\text {bath }}\right)+\frac{G_{\text {Kapitza+Stycast }}}{C_{\text {TES-p }}}\left(T_{\text {Stycast }}-T_{\text {TES-p }}\right) \\
\frac{d T_{\text {TES-e }}}{d t} & =\frac{G_{\text {e-p }}}{C_{\text {TES-e }}}\left(T_{\text {TES-p }}-T_{\text {TES-e }}\right)+\frac{V_{\text {op }}^{2}}{C_{\text {TES-e }} R_{\text {TES }}\left(T_{\text {TES-e }}\right)}
\end{aligned}
$$




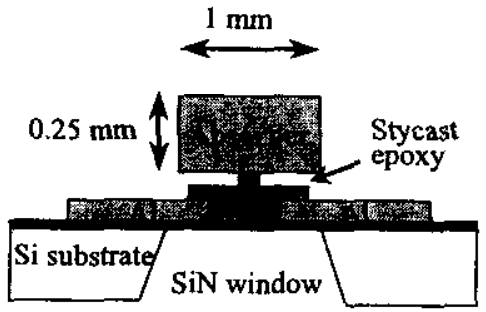

(a)

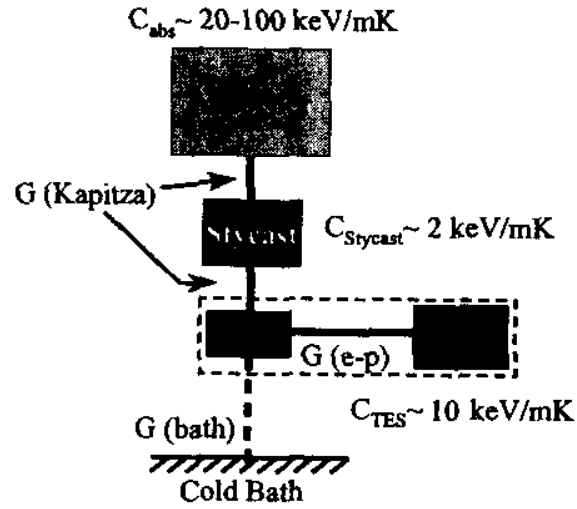

(b)

Figure 1. Schematic diagram of the composite TES microcalorimeter, showing the SiN window, the TES, the Al superconducting leads, and the absorber glued on the TES with Stycast epoxy(a). The corresponding thermal diagram is also shown (b).

temperature. Apart from $R_{\mathrm{TES}}(T)$ and $G_{\text {bath }}$ all parameters can be obtained from literature. $R_{\mathrm{TES}}(T)$ (and thus $\alpha \equiv T / R \cdot d R / d T)$ and $G_{\text {bath }}$ can be derived from the IV curves as a function of bath temperature. ${ }^{10}$

For a $0.25 \mathrm{~mm}^{3} \mathrm{Sn}$ absorber and typical operating conditions of $T_{\mathrm{c}}=100 \mathrm{mK}, T_{\text {bath }}=70 \mathrm{mK}, R_{\mathrm{op}}=0.1 \Omega$, and $\alpha=600$ the model predicts a maximum pulse height of $5 \mu \mathrm{A}$ for a $60 \mathrm{keV}$ gamma-ray photon, a signal rise time of $74 \mu \mathrm{s}$ and a signal decay time of $1.4 \mathrm{~ms}$. The decay time is mainly determined by the time it takes for the heat to leave the absorber $\left(C_{\mathrm{abs}} / 0.5 G_{\text {Kapitza }}+\right.$ Stycast $\left.=1.3 \mathrm{~ms}\right)$. Electrothermal feedback does not shorten the signal as the detector is operated in bolometric mode due to the glue bottleneck. The rise time is limited by the response time of the Stycast epoxy $\left(C_{\text {Stycast }} / G_{\text {Kapitza+Stycast }}=50 \mu \mathrm{s}\right)$ and the electron system (which is determined by the electrothermal feedback).

\section{EXPERIMENTAL SETUP}

The devices are measured in an adiabatic demagnetization refrigerator (ADR) capable of reaching a base temperature of $60 \mathrm{mK}$. The TES is voltage biased using a $10 \mathrm{~m} \Omega$ shunt resistor. The current through the TES is measured with a Hypres model \#30 single-stage 208 series SQUID-array. The typical SQUID noise is less than $10 \mathrm{pA} / \sqrt{\mathrm{Hz}}$.

\section{RESPONSE OF A CALORIMETER WITH TIN ABSORBER}

We have verified the validity of the model and the model parameters given in section 4 with the gamma-ray response of Sn-TES8, a gamma-ray calorimeter with a $0.25 \mathrm{~mm}^{3} \mathrm{Sn}$ absorber and a $T_{c}=100 \mathrm{mK}$. This specific detector has an excellent energy resolution of $70 \mathrm{eV}$ FWHM at $60 \mathrm{keV} .^{10}$ Figure 2 shows the response to the $59.54 \mathrm{keV}$ gamma-ray photons from an ${ }^{241} \mathrm{Am}$ gamma-ray source. Also shown as a dotted line is the pulse shape as predicted by the model using only material properties data from literature except for $G_{\text {bath }}=0.28 \mathrm{nW} / \mathrm{K}$ and $\alpha=600$ (at the operating resistance of $R_{\mathrm{op}}=96 \mathrm{~m} \Omega$ ), which have been derived from the IV curves as a function of bath temperature. ${ }^{10}$, The $R_{\text {TES }}(T)$ has been approximated as a linear function.

Considering the uncertainties in the material properties data, especially those of the Stycast epoxy, the model can predict the pulse shape and height very well. The measured pulse height is about $10 \%$ higher than predicted. The rise time of the pulse is $95 \mu \mathrm{s}$, which compares reasonably well with the $79 \mu$ s rise time from the model. The fall times are also slightly off, the measured $1 / e$ time between $80 \%$ and $20 \%$ is $1.0 \mathrm{~ms}$, whereas the model predicts 1.4 ms. The kinks in the tail of the measured pulse shape are due to irregularities in the $R_{\operatorname{TES}}(T) .^{10}$ Since the model uses a linear approximation for $R_{\text {TES }}(T)$, the simulation does not show the kinks. 


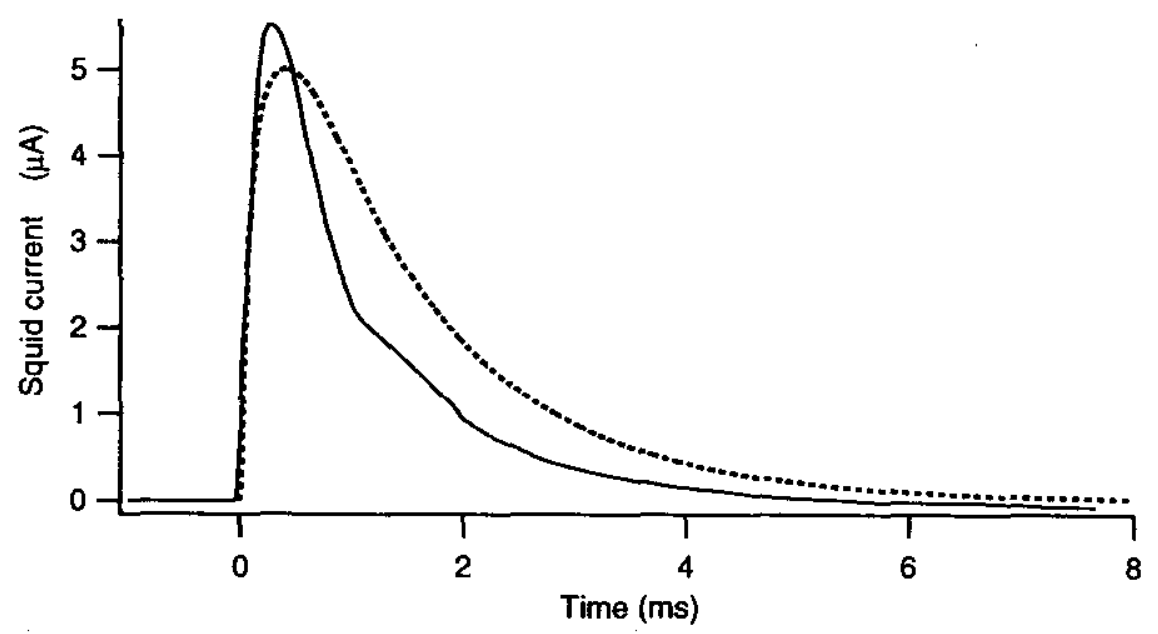

Figure 2. Response of Sn-TES8 to $60 \mathrm{keV}$ gamma-ray pulses. The dotted line shows the predicted response from the model using only material properties data from literature and parameters derived from the IV-curves as a function of bath temperature.

\section{COMPARISON BETWEEN DIFFERENT SUPERCONDUCTING ABSORBERS}

Although the energy resolution of $70 \mathrm{eV}$ FWHM at $60 \mathrm{keV}$ using a microcalorimeter with a $0.25 \mathrm{~mm}^{3} \mathrm{Sn}$ absorber is very encouraging, the absorption efficiency of the $250 \mu \mathrm{m}$ thick Sn absorber decreases rapidly at higher gammaray energies. We have therefore fabricated microcalorimeters with higher-Z superconductors (or lower phonon heat capacity). $\mathrm{Ta}(99.98 \%), \mathrm{Mo}(99.98 \%), \operatorname{Re}(99.99 \%)$ and $\mathrm{Pb}(99.999 \%)$ rolled foils have been purchased from Electronic Space Products International (ESPI). All absorbers have been cut to the same size $\left(1 \times 1 \times 0.25 \mathrm{~mm}^{3}\right)$. They have been glued to TES multilayers from the same production run with $T_{c}=90 \mathrm{mK}$. We have also manufactured another detector with a Sn absorber. The $99.99 \%$ pure Sn foil was purchased from Goodfellow Corporation.

In this paper we present our first measurements of the detectors with a $\mathrm{Pb}$, Ta and $\mathrm{Sn}$ absorber (PbTES1, TaTES1 and SnTES12). Figure 3 shows the response of the calorimeters to $60 \mathrm{keV}$ gamma-rays. The pulse height of SnTES12 is only $1.3 \mu \mathrm{A}$, compared to $5.5 \mu \mathrm{A}$ for SnTES8 shown in the previous section. The rise time is also much longer: $210 \mu$ s compared to $80 \mu$ s previously. The most likely cause for the differences is an inconsistency in the glueing process, since that would affect both the signal rise time and the pulse height. The response of the microcalorimeter with the $\mathrm{Pb}$ absorber is in good agreement with the model. The relatively slow rise time and small pulse height are due to the large heat capacity of the $\mathrm{Pb}$ absorber. The measured pulse shapes for the calorimeter with the Ta absorber differs significantly from the prediction. The measured pulse height is lower and the decay time is longer than predicted, which is indicative of a higher heat capacity. This might be due to interstitial hydrogen in the Ta absorber, ${ }^{19}$ which can be removed by degassing the absorber under vacuum. ${ }^{20}$

Figure 4 shows a plot of the microcalorimeter responses to the $60 \mathrm{keV}$ gamma-rays on long time scales. The pulses have been averaged to increase the signal to noise ratio in the tails. Special care has been taken to reject all pulses that contained any gamma-ray or X-ray events in the tail, including the many $\mathrm{Cu} \mathrm{K}-\alpha \mathrm{X}$-rays $(8 \mathrm{keV})$ that are produced by gamma-rays that are absorbed in the copper heat shield surrounding the detector. The necessity of careful pile-up rejection is indicated by the upper dotted line in Fig. 4, which shows the averaged pulse of the calorimeter with the Sn absorber without pile-up rejection in the tail. A typical pulse without pile-up events is shown as a dotted line for the calorimeter with the $\mathrm{Pb}$ absorber. This unaveraged pulse demonstrates clearly that the averaged pulses do not contain any significant effect from pile-up events. The tails are therefore purely a thermalization effect in the detectors. The tails have been fitted with a double exponential. The thermalization times for the $\mathrm{Pb}$ and $\mathrm{Ta}$ absorbers are, respectively, 29 and $31 \mathrm{~ms}$. The thermalization time for the $\mathrm{Sn}$ absorber is significantly longer (77 $\mathrm{ms}$ ).

The energy resolution of SnTES12 is $200 \mathrm{eV}$ FWHM at $60 \mathrm{keV}$ using optimal digital filtering. ${ }^{21}$ This result is consistent with a factor of three lower pulse height compared to SnTES8. The energy resolution of PbTES1 has not yet been fully optimized with respect to bias point and filtering. Using a gaussian shaping filter with a time constant 


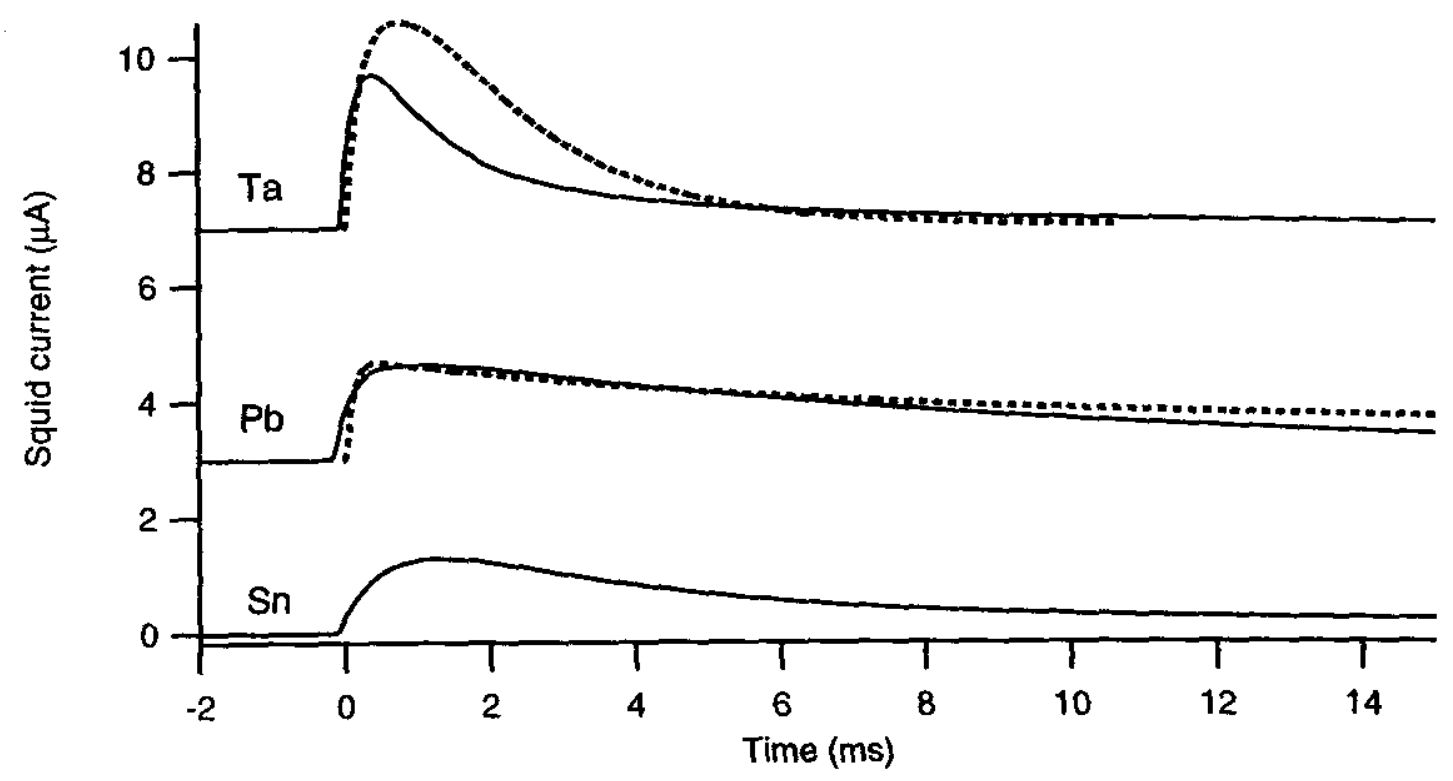

Figure 3. Averaged response of microcalorimeters with a $\mathrm{Ta}, \mathrm{Pb}$ and $\mathrm{Sn}$ absorber to $60 \mathrm{keV}$ gamma-ray pulses. The dotted line shows the predicted response from the model using only material properties data from literature and parameters derived from the IV-curves as a function of bath temperature.

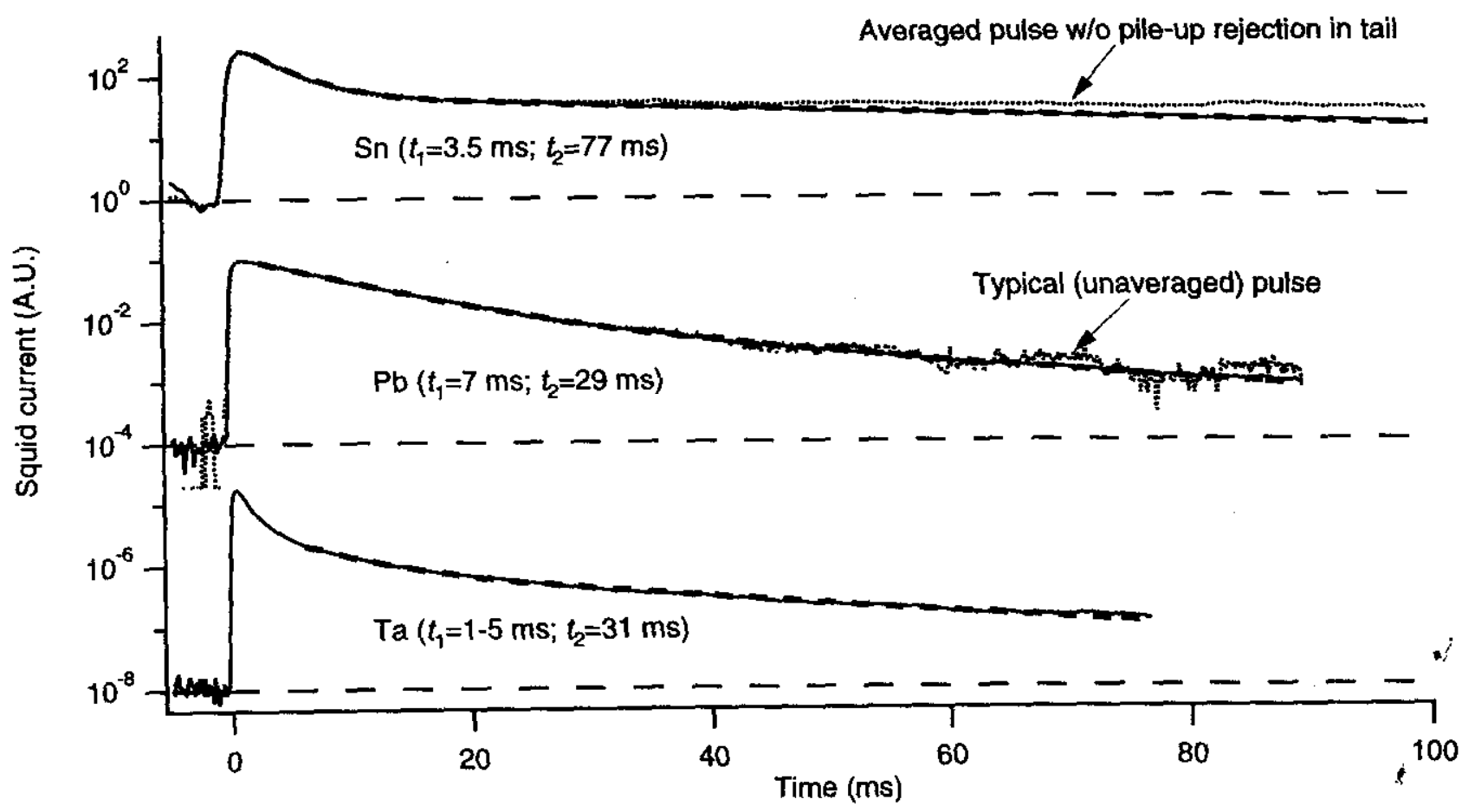

Figure 4. Logarithmic plot of the average pulse shapes of several microcalorimeters due to absorption of $60 \mathrm{keV}$ gamma-ray photons, showing the thermalization properties of the absorbers. The thick dashed lines are double exponential fits to the tail. The dotted line for the calorimeter with the $\mathrm{Sn}$ absorber shows the average response without careful pile-up rejection in the tail. The dotted line for the calorimeter with the $\mathrm{Pb}$ absorber is a typical unaveraged pulse. 
of $750 \mu \mathrm{s}$, the energy resolution is $500 \mathrm{eV}$ FWHM at $60 \mathrm{keV}$. The noise voltage after filtering was $220 \mathrm{eV}$ FWHM for this particular measurement. Based upon our previous experience, we expect that optimization of the bias point and optimal filtering may improve the energy resolution with at least a factor of two.

The calorimeter with the Ta absorber showed a very broad peak of $2 \mathrm{keV} \mathrm{FWHM} \mathrm{(baseline} \mathrm{noise:} 100 \mathrm{eV}$ FWHM) and a correlation between pulse height and rise time. Pulses with faster rise time have a larger pulse height. Such a correlation has not been found in any of the other detectors. Since the phonons themselves will quickly diffuse across the absorber, this can only be caused by a variation in the fraction of energy that is converted into phonons. The thermalization process that takes place in the absorber is very complicated and the phonon distribution that is created depends on many factors including quasiparticle life time and quasiparticle diffusion. There was no drift in pulse height, nor any correlation between pulse height and pretrigger baseline level. Although unlikely, given the relatively fast thermalization in the Ta absorber, the latter correlation would have pointed to a dependence of the pulse height on the quasiparticle density before absorption of the photon (as discussed in Sect. 3.2). Another possibility is that the quasiparticle life time and thus the pulse height depends on the position of photon impact. The observed correlation with rise time would then reflect differences in quasiparticle recombination rate during the initial relaxation. We plan to further explore this issue by studying Ta absorbers with different impurity levels, including single crystals. An additional quasiparticle trapping layer ${ }^{22}$ might also reduce the position dependence of the response.

\section{CONCLUSIONS}

We have demonstrated an energy resolution of $70 \mathrm{eV} \mathrm{FWHM} \mathrm{for} 60 \mathrm{keV}$ gamma-rays using a $1 \times 1 \times 0.25 \mathrm{~mm}^{3} \mathrm{Sn}$ absorber glued with epoxy to a $\mathrm{Mo} / \mathrm{Cu}$ multilayer TES. The response of this microcalorimeter is consistent with predictions based on material properties and parameters derived from the IV curves of the TES as a function of bath temperature. The glueing process appears to be critical for a good detector performance.

We have started an extensive study to optimize the quantum efficiency of the gamma-ray calorimeter by fabricating microcalorimeters using superconducting absorbers with a higher stopping power or lower heat capacity. Of the two absorber materials tested, $\mathrm{Pb}$ appears to be more promising than $\mathrm{Ta}$. We plan to explore other superconductor materials, such as Re and Mo, as well as different types of Ta absorbers, including single crystals.

\section{ACKNOWLEDGMENTS}

Jan Batteux is acknowledged for excellent experimental and technical support. This work was performed under the auspices of the U.S. Department of Energy by University of California Lawrence Livermore National Laboratory under contract No. W-7405-Eng-48, with support from NASA Constellation-X Technology Development Program under interagency agreement S10256-G and the NASA High Energy Astrophysics Supporting research and Technology Program under interagency agreement W-19, 121.

\section{REFERENCES}

1. S. H. Moseley, J. C. Mather, and D. McCammon, "Thermal detectors as x-ray spectrometers," J. Appl. Phys., 56, pp. 1257-1262, 1984.

2. K. D. Irwin, "An application of electrothermal feedback for high resolution cryogenic particle detection," Appl. Phys. Lett., 66, pp. 1998-2000, 1995.

3. S. Vitale, G. Gallinaro, and F. Gatti, "Alpha- beta- and gamma-ray detection with microcalorimeters made/with superconducting absorbers," in EUV, X-Ray, and Gamma-Ray Instrumentation for Astronomy III, $\mathrm{O}$. $\mathrm{H}$. W. Siegmund, ed., Proc. SPIE 1743, pp. 368-379, 1992.

4. S. B. Kaplan, C. C. Chi, D. N. Langenberg, J. J. Chang, S. Jafarey, and D. J. Scalapino, "Quasiparticle and phonon lifetimes in superconductors," Phys. Rev. B, 14, pp. 4854-4873, 1976.

5. E. Cosulich, F. Gatti and S. Vitale, "Further results on $\mu$-calorimeters with superconducting absorbers," J. of Low Temp. Phys., 93, pp. 263-268, 1993.

6. F. Gatti, S. Vitale, and A. Barabino, "Superconducting rhenium as absorber for thermal detectors," Nucl. Instr. and Meth. in Phys. Res. A, 315, pp. 260-262, 1992. 
7. C. K. Stahle, D. Osheroff, R. L. Kelly, S. H. Moseley, and A. E. Szymkowiak, "Adapting calorimetric X-ray detectors for Compton scattering experiments performed at high energies," Nucl. Instr. and Meth. in Phys. Res. $A, 319$, pp. 393 -399, 1992.

8. G. Forster, K. Hallatschek, D. Wilhelm, F. Pröbst, W. Seidel, E. Kellner, and F. v. Feilitzsch, "Calorimetric particle detectors with superconducting absorber materials," J. Low Temp. Phys, 93, pp. 219-224, 1993.

9. H. J. Meier, P. Egelhof, S. Han, H.-J. Kluge, D. McCammon, T. Mihara, S. H. Moseley, and C. K. Stahle, "Low temperature detectors for precise Lamb shift measurements on hydrogen-like heavy ions," in Proc. of the 7th International Workshop on Low Temperature Detectors, S. Cooper, ed., pp. 213-214, Max Planck Institute of Physics, Munich, Germany, 1997.

10. D. T. Chow, A. Loshak, M. L. van den Berg, M. Frank, T. W. Barbee Jr., S. E. Labov, "High-resolution gammaray spectrometers using bulk absorbers coupled to $\mathrm{Mo} / \mathrm{Cu}$ multilayer superconducting transition edge sensors," to be published in Hard X-Ray, Gamma-Ray, and Neutron Detector Physics II, R.B. James and R.C. Shirato, eds., Proc. SPIE 4141, 2000.

11. E. Silver, G. Tucker, S. Murray, M. Juda, J. Juda, N. Madden and J. Beeman, "X-ray microcalorimetry research and development," in Proc. of the HTXS workshop, H. Tananbaum, N. White, P. Sullivan, eds., pp. 458-468, Harvard-Smithsonian Center for Astrophysics, Cambridge, 1996.

12. A. Bleile, P. Egelhof, H.-J. Kluge, U. Liebisch, D. McCammon, H. J. Meier, O. Sebastián et al., "Lowtemperature X-ray detectors for precise Lamb shift measurements on hydrogen-like heavy ions," Nucl. Instr. Meth. in Phys. Res. A, 444, pp. 488-491, 2000.

13. A. Alessandrello, J. W. Beeman, C. Brofferio, O. Cremonesi, E. Fiorini, A. Giuliani, E. E. Haller et al., "High energy resolution bolometers for nuclear physics and X-ray spectroscopy," Phys. Rev. Lett., 82, pp. 513-515, 1999.

14. C.A. Swenson, "Linear thermal expansivity (1.5-300 K) and heat capacity (1.2-90 K) of Stycast 2850FT," Rev. Sci. Instrum., 68, pp. 1312 1315, 1997.

15. A. C. Anderson and R. E. Peterson, "Selection of thermal bonding agent for temperatures below 1K," Cryogenics, 10, pp. 430-433, 1970.

16. J. R. Olson, "Thermal conductivity of some common cryostat materials between 0.05 and $2 \mathrm{~K}$," Cryogenics, $\mathbf{3 3}$, pp. 729-731, 1993.

17. F. C. Wellstood, C. Urbina, and J. Clarke, "Hot-electron effects in metals," Phys. Rev. B, 49, pp. 5942-5955, 1994.

18. D. T. Chow, M. A. Lindeman, M. F. Cunningham, M. Frank, T. W. Barbee Jr., and S. E. Labov, "Gamma-ray spectrometers using a bulk $\mathrm{Sn}$ absorber coupled to a $\mathrm{Mo} / \mathrm{Cu}$ multilayer superconducting transition edge sensor," Nucl. Instr. Meth. in Phys. Res. A, 444, pp. 196-200, 2000.

19. G.J. Sellers, M. Paalanen and A.C. Anderson, "Anomalous heat capacity of superconducting vanadium," Phys. Rev. B, 10, pp. 1912-1915, 1974.

20. K. Faber and H. Schultz, "Hydrogen contamination in Ta and Nb following UHV-degassing," Scr. Metall., 6, pp. $1065-1070,1972$.

21. A. E. Szymkowiak, R. L. Kelley, S. H. Moseley, and C. K. Stahle, "Signal processing for microcalorimeters," J. Low Temp. Phys., 93, pp. 281-285, 1993.

22. N.E. Booth, "Quasiparticle trapping and the quasiparticle multiplier," Appl. Phys. Lett., 50, pp. 293-295, 1987. 
